\title{
HF accelerated electron fluxes, spectra, and ionization
}

\author{
Herbert C. Carlson ${ }^{1}$ and Joseph B. Jensen ${ }^{1,2}$ \\ ${ }^{1}$ Physics Dept./CASS, Utah State University, Logan UT 84322, USA \\ ${ }^{2}$ Physics Dept., University of New Hampshire, Durham, NH 03824, USA
}

Abstract: Wave particle interactions, an essential aspect of laboratory, terrestrial and astrophysical plasmas, have been studied for decades by transmitting high power HF radio waves into Earth's weakly ionized space plasma, to use it as a laboratory without walls. Application to HF electron acceleration remains an active area of research (Gurevich, 2007) today. HF electron acceleration studies began when plasma line observations proved (Carlson et al, 1982) that high power HF radio wave-excited processes accelerated electrons not to $\sim \mathrm{eV}$, but instead to 100 times thermal energy $(10 \mathrm{~s}$ of eV), as a consequence of inelastic collision effects on electron transport.

Gurevich et al (1985) quantified the theory of this transport effect. Merging experiment with theory in plasma physics and aeronomy, enabled prediction (Carlson, 1993) of creating artificial ionospheres once $\sim \mathrm{GW}$ HF effective radiated power (ERP) could be achieved. Eventual confirmation of this prediction (Pedersen et al 2009, 2010; Blagoveshchenskaya et al, 2009) sparked renewed interest in optical inversion to estimate electron spectra in terrestrial (Hysell et al, 2014) and planetary (Simon et al, 2011) atmospheres. Here we present our unpublished optical data, which combined with our modeling, lead to conclusions that should meaningfully improve future estimates of the spectrum of HF accelerated electron fluxes. Photometric imaging data can significantly improve detection of emissions near ionization threshold, and confirm depth of penetration of accelerated electrons many $\mathrm{km}$ below the excitation altitude. Comparing observed to modeled emission altitude shows future experiments need electron density profiles to derive more accurate HF electron flux spectra.

Key words HF electron acceleration, optical emissions, HF ionization

\section{Introduction}

When ionospheric modification experiments were first planned, thought was in terms of heating the electron gas by derivative absorption heating: each half cycle of the radio frequency (rf) wave it would give rf energy to ambient 
electrons, and each other half cycle the electrons would return the rf energy absent collisions. An electron suffering a collision deviating its cyclical motion, cannot return all the energy it received, so that energy is lost to random motion, i.e. heat into the electron gas. Near the height of reflection of the HF radio wave (transmitted at a frequency below the maximum ionospheric plasma frequency), as the wave slows down, many collisions occur, and electron gas heating results-hence the terminology "heating experiments".

When the electron gas is heated, the altitude profile of the plasma expands away from the peak, mostly upwards expansion, reducing the peak density and in effect forming an HF radio wave refracting lens. When heating experiments began (Utlaut and Cohen 1971; Gordon et al. 1971) electron gas temperature $\left(T_{e}\right)$ enhancements were sensed by optical techniques (Sipler and Biondi 1972) before incoherent scatter radars were used (Gordon et al. 1971). Unexpected by most, it was soon proven experimentally (Carlson et al. 1972) with theory updated to follow (Perkins et al. 1974) to show that if the HF radio wave power density delivered to the plasma is sufficiently great to alter the internal energy of the electron gas (raise $T_{e}$ ), the associated electric field is sufficiently great to excite plasma instabilities. These high power HF radio waves led not only to a host of plasma wave instabilities, but also to considerable structuring of the plasma over a very broad range of scale sizes (Fialer 1974), as well as wave particle interactions leading to electron acceleration.

Early theory concluded that electron acceleration could raise electron energies from thermal levels $(\sim 0.1 \mathrm{eV}$ background) to order one to a few eV (Fejer, 1977, 1979; Weinstock 1974) but not higher. Photometers fielded to sense optical emissions at $630.0 \mathrm{~nm}$, as they could be excited by suprathermal electrons $>1.96 \mathrm{eV}$, or by Te regions $>\sim 3000 \mathrm{~K}$, and also could sense Te enhancements indirectly by depressing the Te dependent recombination rate, or $557.7 \mathrm{~nm}$ excited by electrons of energy $>4.19 \mathrm{eV}$ were not pressed to look for higher energy excitation lines.

An experiment was then designed to explore whether electron acceleration processes might achieve energies approaching ionization potential (Carlson et al. 1982). That experiment proved beyond doubt that HF induced electron acceleration reached energies above the ionization potential for atomic oxygen and molecular nitrogen, directly detecting plasma line signatures of electrons across the $10-20 \mathrm{eV}$ range. Their plasma line data showed a supra-thermal electron flux relatively flat over the $10-20 \mathrm{eV}$ range, no strong non-linear power dependence on $\mathrm{HF}$ power, and continuing presence for hours.

The observations demonstrated the presence of significant electron fluxes to energies of at least $20 \mathrm{eV}$. This posed a serious problem for published theories (Fejer, 1977, 1979), which did not accelerate significant fluxes to 
such high energies. The downward electron flux was found to be relatively flat in the $10-20 \mathrm{eV}$ range, and was estimated as rather stable for hours, at 1-2 x $10^{6}$ electrons $\mathrm{cm}^{-2} \mathrm{~s}^{-1} \mathrm{eV}^{-1}$, or 2-4 $\times 10^{7}$ electrons $\mathrm{cm}^{-2} \mathrm{~s}^{-1}$, carrying $2-4 \mathrm{x}$ $10^{8} \mathrm{eV} \mathrm{cm}^{-2} \mathrm{~s}^{-1}$ energy flux (or 0.34.6 $\mathrm{PW} \mathrm{me*).} \mathrm{This} \mathrm{estimate} \mathrm{is} \mathrm{confirmed} \mathrm{by} \mathrm{coincident} \mathrm{independent} \mathrm{airglow}$ observations showing a $20 \mathrm{R}$ enhancement of $630.0 \mathrm{~nm}$, airglow, due to $\mathrm{O}\left({ }^{1} \mathrm{D}\right)$ impact excitation by this flux of electrons. The thermal electron gas in the $250-280 \mathrm{~km}$ region was enhanced by about $150 \mathrm{~K}$ too low for thermal excitation.

Carlson et al. (1982) highlighted that due to the elastic and inelastic cross sections in the thermosphere at the altitudes in question, the suprathermal electrons have a mean-free path of several km and suffer about ten elastic collisions before each inelastic collision. Any acceleration mechanism will see 'loss-cones' rapidly fill in. Any flux initially directed down will produce a steady-state flux in both directions, about one-third of the total flux at any level being backscattered up towards the source region. The plasma line enhancements (suprathermal electron fluxes) reported here persisted for over $2 \mathrm{~h}$ on this night. The steady electron fluxes contradict strong nonlinear dependence on the HF power density (theoretically predicted 1000 fold flux enhancements for a four fold increase in HF power density was violated relative constancy while the range squared variations in HF power density would have been large simply from the changes in the HF reflection altitude). The paper suggested that inelastic collisions would allow accelerated electrons to repeatedly revisit the thin electron acceleration region before filling the ionospheric/ thermospheric volume a few thermospheric scale heights thick $(\sim 100 \mathrm{~km})$.

Within three years a theory was developed (Gurevich et al. 1985) employing this suggestion in a plasma physics formalism, to explain $\mathrm{HF}$ acceleration of electrons to several $10 \mathrm{~s} \mathrm{eV}$, well above ionization potential. Rose et al. (1985) published results from firing rockets through the Tromso HF heater volume, to measure in situ many HF excited properties including HF accelerated electrons. They published that HF excited electrons were excited to above $9 \mathrm{eV}$, a threshold below ionization threshold, but within the context of evidence for a flat electron spectrum in the $10-20 \mathrm{eV}$ range, meaningful.

With experiment and theory in agreement that ionization could be and was produced, the next question was how significant that ionization production could be. Combining the incoherent scatter constraints established for the altitude spread of the electron flux, and its magnitude and relative steadiness, allowed a first estimate of efficiency of ionization production, and led to a prediction and proposal (Carlson 1987) to build an HF heater capable of producing ionization competitive with that by the sun. That proposal was selected for funding by AFOSR in 1987, 
but a congressional budget cut intervened before the authorization phase and all AF basic research new starts were canceled that year. The concept was eventually published (Carlson 1993) including the quantitative prediction that a 1 GW ERP (effective radiated power) could create ionospheres competitively with the sun. By 2009 other HF heating facilities were in operation approaching this ERP (Blagoveshchenskaya et al. 2009; Pedersen et al., 2009, 2010).

That the ionization production mechanism was HF electron acceleration producing a suprathermal electron spectrum exceeding $20 \mathrm{eV}$, pressed the need for useful experimental constraints on definition of this spectrum, to help to guide theory to better predictions. A more practicable way than incoherent scatter radars and rockets, to sense/monitor HF accelerated electrons to energies $\sim 10 \mathrm{eV}$ or more, was highly desirable. Observation of a range of optical emissions of excitation thresholds $>\sim 10 \mathrm{eV}$ was a very attractive way to go (e.g. Bernhardt et al. 1989 and other paper therein coauthored by Gustavsson, Eliasson, and Hysell). The concept was: each different optical emission line intensity could represent a different integral of particle energies under the impact cross-section curve, weighted by the relative neutral number densities encountered along the electron flux path from source altitude to sink. For a thin electron acceleration region at an optically thin source region, much of the up-going flux would escape to the conjugate hemisphere while the down-going flux would penetrate to its altitude of unity optical depth, where it would produce most optical emission (and ionization) within about a neutral scale height of that altitude. For an electron acceleration source region in an optically thick altitude, the spread would be closer to and about the acceleration height.

With optical instrumental capabilities improving, through photometers and then photometric imaging, plus motivation from artificial ionization production, there is powerful impetus to explore the potential of differential excitation cross-sections for optical emissions, for estimating the magnitude and shape of suprathermal electron spectra. That is our focus here.

\section{Observations}

The experiment we now present the results from was conceived and designed to provide a complimentary independent confirmation of the presence of $\mathrm{HF}$ accelerated electrons to energies above $10 \mathrm{eV}$, i.e. approaching ionization potential, at the mid to low latitude location of Arecibo (18.3 $3^{\circ}$ geographic north, $\mathrm{L}$ value $\left.\sim 1.5\right)$. [Note it 
has since been theoretically predicted (Gurevich et al. 2002) and experimentally confirmed at all high latitude HF facilities (Pedersen and Carlson 2001; Kosch et al., 2000; Gurevich et al. 2001, 2002, 2005), that there is a significant difference between high vs lower latitude high power HF effects, due to prediction/confirmation of what has come to be known and embraced as the magnetic zenith effect (see review by e.g. Gurevich et al. 2005). Effects of high power HF transmission at high latitudes can also be significantly amplified by HF operation at multiples of the electron gyro frequency (Djuth et al. 2005; Blagoveshchenskaya et al. 2009; Pedersen et al., 2010, etc.), which currently are not accessible to the Arecibo HF facility.

The April 1988 optical observations we present here were gathered at $630.0 \mathrm{~nm}, 557.7 \mathrm{~nm}$ and $777.4 \mathrm{~nm}$ over the Arecibo Observatory. These have electron impact excitation thresholds respectively of 1.96, 4.19, and $10.74 \mathrm{eV}$. There has been some confusion in the literature as to "whether the $630 \mathrm{~nm}$ HF enhancements are due to thermal or supra-thermal electrons." As a general answer, they can be either or both, depending on the electron density profile (Carlson, 1996). More specifically, to get thermal excitation of $630 \mathrm{~nm}$ emission requires an electron gas temperature above $-2700 \mathrm{~K}$. For a given HF heating rate, thermal balance leads to an electron temperature determined by the cooling rate of the electron gas. This in turn is dependent on the number of electrons times the number of ions, to which they loose energy in collisions; the electron gas cooling rate depends on the square of the electron density. (See e.g. Mantas et al. 1981 for a detailed discussion of electron gas thermal balance, and the key role of thermal conductivity, within a context directly relevant to this discussion.) If the electron density is near $10^{6} \mathrm{~cm}^{-3}$ at the height of HF reflection, the F region electron gas is very tightly coupled by to the ion gas, by electron-ion collisions, and its temperature remains close to that of the ions, too low for $630 \mathrm{~nm}$ thermal excitation. If the electron density is near $10^{5} \mathrm{~cm}^{-3}$ at the height of HF reflection, the electron gas cooling-rate is 100 times lower: The electron gas is, in effect, thermally insulated from the local (ion) heat sink, and its temperature can rise very significantly above that of the ions, and be a good candidate to thermally excite 630 $\mathrm{nm}$. For example, at HAARP heating at $\sim 3 \mathrm{MHz}$ away from any resonances will give large $\mathrm{T}_{\mathrm{e}}$ enhancements, at Arecibo heating at high HF frequencies will not.

The data we present were collected at the Arecibo Observatory on April 20, 1988, between 02:00 - 03:00 AST (Atlantic Standard Time or local time). The heater was cycled on/off to permit subtraction of background intensities based on the off vs on cycle. The HF transmitter was on for 2 minutes of each four-minute cycle and off for the other two, operating at $5.1 \mathrm{MHz}$ with $100 \mathrm{KW}$ from each of the four HF transmitter sub-units, into the rectangular 
dipole field north of the Arecibo Observatory. We have no reason to anticipate measurable thermal excitation. The data in Figure 1 is from the second minute on in the four minute cycle 02:24-02:28 AST. Although the natural relaxation time for the $\mathrm{O}\left({ }^{1} \mathrm{D}\right)$ state is near two minutes, quenching makes a 30-50 s time constant typical for Arecibo HF heating experiments. We used an S-20 extended-red photocathode to feed our ASIP II.

It is key that these data were collected using a new all sky imaging photometer (ASIP). We found that within a single image, the HF electron-impact component of airglow stood out against the background airglow with sufficient clarity that identification was most readily found from subtracting the surrounding 2-D flat field background intensity relative to the intensity enhanced within the clearly defined HF enhanced region. In Figure 1, this can be seen by looking at the intensity contours within the ASIP image. Figure 1 is a reproduction of the original raw data with no processing other than four progressively higher degrees of smoothing over adjacent pixels in the raw image. One purpose of this was first to experimentally verify that there was no "Magnetic Zenith effect" at the Arecibo latitude, as theoretically expected at this low latitude, in contrast to such effects now known at high latitudes. The second purpose was to test how much smoothing would be needed to get good noise statistics on the HF electron impact excited component of the image, to separate it from the background. So doing within an individual image eliminates problems of varying intensity from one time to the next, a significant improvement reducing noise or bias.

We then repeated this step for the $557.7 \mathrm{~nm}$ and $777.4 \mathrm{~nm}$ images, to get the clearly defined contours of airglow enhancement, within Figure 2a. For the merged Figure 2a, we needed two further steps:

First, the contours were so well defined (seen in Figure 2), that we could align the contours for each emission line $(630.0,557.7,777.4 \mathrm{~nm})$ on each other closely enough to define a horizontal (latitude/longitude) displacement of the contours relative to one another. Note that the center peak-intensity-contour in each case aligns well when all lower intensity level contours match best, so all emission contours are consistent with the expectation of excitation electron trajectories being largely confined to move along the magnetic field. Reassuringly we found the center of each set of contours was in the common plane of the magnetic declination.

Second, this conforms to the physical expectation that all emissions should be centered on a common magnetic field line, so the only unknown is reduced to the altitude of the center of gravity of the emission volume. Therefore, we drew a line with the dip angle of the Arecibo magnetic field line at the time these data were taken (note this is a moving target model for which that can be tracked on the web). With the only remaining unknown as the altitude of 
the center of gravity of the emission contours, within this one degree of freedom we slid the contours up or down the magnetic field line to center the contours about that line. Putting the field line through the center of one emission line then defined the altitude of the other two. As expected the 630.0 emission is at the highest altitude (near the source region). We then find the 557.7 emission closely below it, and the 777.4 emission lowest in altitude. This is consistent with one's first-reaction intuition in that harder particles penetrate more deeply into the atmosphere than softer particles. We shall discuss this further in the modeling section.

We plot both this Arecibo data and the HAARP data on a common diagram, Figure 2, to help us remember to "keep the end in mind from the beginning." Our goal is to use optical emissions as a tool to help understand the spectrum of the HF accelerated electrons, with particular emphasis on relevance to production of significant ionization. We have long known some ionization is produced (e.g. Carlson et al. 1982), the issue here is whether significant ionization is produced, relative to that by the sun (Pedersen et al., 2009, 2010).

Figure $2 \mathrm{~b}$ shows data from HAARP, of $557.7 \mathrm{~nm}$ volume emission rate, on an altitude vs horizontal distance scale. It shows only $557.7 \mathrm{~m}$ emission intensity, so the color scale represents different degrees of $557.7 \mathrm{~nm}$ intensity. This is from a publication (Pedersen et al. 2010) which also shows ionization production peaks nominally collocated with the $557.7 \mathrm{~nm}$ emission peaks, so Figure $2 \mathrm{~b}$ color contours of most significant electron impact emission excitation are nominally indicative of regions of most significant ionization production. In this sense on Figure 2a, the $777.4 \mathrm{~nm}$ emission (violet), is at energies $(>10.7 \mathrm{eV})$ closest to the ionization potential for atomic oxygen $(13.6 \mathrm{eV})$. We can now move forward to the modeling component of the study enabling our conclusions.

\section{Updating of Mantas model}

The initial plasma line work estimating HF suprathermal electron fluxes, used a software package described in Carlson et al. (1982 and references therein) and from which our Figure 3 here reproduces their Figure 3. Prof. Mantas kindly worked with us recently at USU to enable us last year to revitalize this software, re-compiling to run on today's machines. For consistent baselines, changing only one thing at a time, we have run these programs with the same cross-sections/rates as in 1982, so comparisons can initially remain in a common frame or reference. Comparison of our data herein, with output from these models below, is quite instructive well beyond this data set alone. It offers value for optimizing future data collection as well as interpretation of past and future data. 
Model runs in Figures 3, 4, 5 all use an MSIS thermosphere model input, with very minor smoothing to keep both neutral density and its altitude derivative smooth for purposes of computer program stability. The actual measured electron density profile was included for Figure 3 (Carlson et al. 1982), here in the absence of a measured electron density profile we omitted that input to the program for Figures 4 and 5 . Recall we are comparing profiles of optical emission from the thermosphere, not electron densities or plasma lines in the ionosphere.

As done in Carlson et al. (1982), we start with injection of a flat spectrum of electrons within a single thin altitude slab, to represent a thin slab within which HF excited plasma instability processes would produce a thin electron flux source region (as detailed in e.g. Gurevich 2007 and references cited therein). Figure 4 shows the results for a thin altitude source slab of HF accelerated electrons at three altitudes, one at which the thermosphere is: very optically thin $(982 \mathrm{~km})$ far right hand side, intermediate $(505 \mathrm{~m})$ center, and optically thick $(254 \mathrm{~km})$ left hand side of figure. The optically thin case is similar to the model first published by Haslett and Megill (1974), based on their observations at the Platteville heater near Boulder CO, and which is also representative of Arecibo conditions well before "midnight collapse". The up-going flux largely escapes to the conjugate hemisphere and the downgoing flux barely penetrates to $250 \mathrm{~km}$ on this scale. For the HF electron source slab in the optically thick region, most of the flux is absorbed within a neutral scale height on either side of the source region. This latter case is representative of Arecibo conditions with midnight collapse near full descent. The intermediate slab location is indeed intermediate. The only difference between the upper vs lower triple of plots in this Figure 4 is a sufficiently different flux scale, to readily show a fifty-fold range of flux intensities. "Midnight collapse" is a name given to a regular feature of lower mid-latitude ionospheric nighttime behavior, where the ionosphere held up at very high altitudes by neutral winds, falls by $\sim 50-100 \mathrm{~km}$ or more when the neutral winds abate often shortly after midnight (see e.g. Gong et al. 2012).

Figure 5 shows results from calculations with the same updated model as for Figure 4, but now in the form of altitude profiles of steady state flux binned for optical emission of $557.7 \mathrm{~nm}, 777.4 \mathrm{~nm}$, and for electron impact ionization, all for atomic oxygen (thresholds respectively of $\sim 4.2,10.7,13.6 \mathrm{eV}$ ). Figure 3 in 1982 had been for 2 $\mathrm{eV}$ bins of flux flat from $0-20 \mathrm{eV}$, to establish electron acceleration up to at least $20 \mathrm{eV}$, above thermosphere ionization thresholds. By now theory has advanced to make the more relevant question where between 1-100 eV does the electron spectrum essentially cut off, so in Figure 5 we work in $10 \mathrm{eV}$ bins of flux flat from 1-100 eV. One finds the essential information for insight is well captured by the $70-80 \mathrm{eV}$ examples, where we therefore choose to 
stop showing examples.

Perhaps the most straight-forward set of plots to follow is the optically thin case in Figure 5A, with the singlealtitude electron-flux-source at $982 \mathrm{~km}$. First, look only at $10 \mathrm{eV}$ wide bins. For 1-10 eV, only 557.7 nm shows any emission, because the other two thresholds are $>10 \mathrm{eV}$. Ten eV bins of 20-30 eV or higher all follow a common well defined pattern where more energetic particles penetrate deeper (including that profiles for production of $\mathrm{O}^{+}$ penetrate more deeply/ generate higher yields at each height than $777.4 \mathrm{~nm}$, and $777.4 \mathrm{~nm}$ likewise relative to 557.7 $\mathrm{nm})$. The 10-20 eV bin is mixed because of secondary/cascading-energy electron fluxes. Next examine the integral plots of 1-20, 1-40,1-60, and 1-80 eV plots; they have a clearly different pattern in that the 557.7 nm curve (in sharp contrast) always shows $557.7 \mathrm{~nm}$ peaking below $777.4 \mathrm{~nm}$ profiles, not above. This is because of the cascade and build-up of secondary electrons to lower energies. We shall say more about this in the discussion section.

Figure 5B is the same as Figure 5A, except for the optically thick case (electron source flux flat 1-100 eV, in a single altitude slab at $254 \mathrm{~km}$ ). These optically thick cases exhibit the very same main qualitative and semiquantitative features. For all $10 \mathrm{eV}$ bins $20-30$ and above, the $\mathrm{O}^{+}$production profile always penetrates more deeply than $777.4 \mathrm{~nm}$, the $777.4 \mathrm{~nm}$ profile always penetrates more deeply than the $557.7 \mathrm{~nm}$ profile. The profiles for the integrals spanning $1-20,1-30,1-40, \ldots, 1-80 \mathrm{eV}$ the 557.7 and 777.4 penetration depth reverses, and $557.7 \mathrm{~nm}$ profiles are all lower in altitude, not higher, than for the only $10 \mathrm{eV}$-wide bin profiles. The same explanation applies, the secondary electrons cascading down to below $20 \mathrm{eV}$ energy add up to strongly enhance the $<20 \mathrm{eV}$ part of the profile.

The intermediate case between Figures 5A and 5B (not shown here for brevity of presentation) showed the same pattern for that case of an electron source region in a single altitude slab intermediate between an optically thin and thick case. Also common to optically thin through thick cases, the emission profiles for $10-20 \mathrm{eV}$ are at virtually the same height for 777.4 and $557.7 \mathrm{~nm}$, so it is only the contribution for electron fluxes or energy $<10 \mathrm{eV}$ that accounts for this 777.4 vs 557.7 penetration height reversal. We will revisit this as well in the Discussion section.

Note that Figure 4 here is for the integral flux 1-100 eV only. Similar plots were also done for a series of ten 10 eV wide bins, to see how a single $10 \mathrm{eV}$ energy bin would cascade and fill in lower energies with its secondary, tertiary, etc. electrons. Once the $10 \mathrm{eV}$ bin reached $40-50 \mathrm{eV}$ and above, there was an $\sim 20 \mathrm{eV}$ discontinuous jump (e.g. a $10 \mathrm{eV}$ wide bin from 50-60 eV, would yield negligible 40-50 eV secondary electrons, but fill in below) (20 eV wide bins had no such gaps). This ties to the idea that for the F region one needs an additional $20 \mathrm{eV}$ to get 
another ionization. (In the E Region it is $\sim 35 \mathrm{eV}$ per ionization pair (Rees and Roble 1986).)

Now we are ready to compare the observed optical emission altitude to those from our model calculations to see what we learn.

\section{Discussion}

Recall the motivation for this research area is to see what we can learn about the HF accelerated suprathermal electron energy spectrum. At a more general level, the goal is to work backwards from measurements of optical emissions at several different wavelengths, to estimate the energy spectrum of electrons present. This is somewhat analogous to the problem of using optical data of the nature to make estimates of auroral particle fluxes (e.g. Strickland et al. 1983), except in their auroral case they could narrow assumptions about the electron spectrum to two models- a Maxwellian and a Gaussian distribution. In this HF field of study we are still early in developing the theory, and need experimental guidance to help its further development (e.g. Gurevich 2007). Gustavsson and Eliasson (2008) used optical emissions to set parameters in a physics based model. Hysell et al. (2014) employed a non-parametric based inversion approach. Sergienko et al. (2012) used a Monte Carlos model for electron transport. These papers give some context for our work here in terms both seeking more realism of conversion of optical observations to suprathermal electron fluxes, and remaining mindful of mutual dependence/independence of assumptions about theory. We will address a missing term in the equation and observations to estimate its magnitude.

To put our $777.4 \mathrm{~nm}$ observations in current observational context, by now the compliment of optical instruments that have been fielded span: red-line emission at $630 \mathrm{~nm}$ associated with the radiative relaxation of the $\mathrm{O}\left({ }^{1} \mathrm{D}\right)$ state with excitation threshold of $1.96 \mathrm{eV}$; green-line emission at $557.7 \mathrm{~nm}$ associated with the radiative relaxation of the

$\mathrm{O}\left({ }^{1} \mathrm{~S}\right)$ state with excitation threshold of $4.19 \mathrm{eV}, 777.4 \mathrm{~nm}$ from the radiative deactivation of the 3p5P state of atomic oxygen with an excitation threshold of $10.74 \mathrm{eV}, 844.6 \mathrm{~nm}$ in the F region mainly from electron impact excitation of atomic oxygen in the 3p3P state, with excitation threshold is $10.99 \mathrm{eV}$, and the blue-line emission at $427.8 \mathrm{~nm}$ associated with electron impact ionization of molecular neutral nitrogen and the subsequent excitation of the B2 state, with excitation threshold of this state is $18.75 \mathrm{eV}$. For production of ionization in the F2 region dominated by atomic oxygen, the ionization potential is $13.62 \mathrm{eV}$. (Note: Only one relevant observation is known to 
date during heating experiments for this (Mutiso et al. 2008), namely $\mathrm{O}^{+} 732-733 \mathrm{~nm}$ emission, found consistent with electron impact ionization. The importance of collisional quenching of $\mathrm{O}\left({ }^{1} \mathrm{D}\right)$ (negligible for the $\mathrm{O}\left({ }^{1} \mathrm{~S}\right)$ state or other prompt emissions in the F region) are well known, and other complications have been long discussed (Rees and Roble 1975); related work still continues (Kalogerakis et al. 2009).) Our focus here will be use of the compliment of wavelengths as they relate to diagnosing suprathermal electron spectra.

In comparing our data with the model profiles, the most striking thing is the discrepancy in the height of the observed vs modeled 777.4 vs $557.7 \mathrm{~nm}$ emission profile. They are opposite to that from the energy integral flux. The altitude of the observed $777.4 \mathrm{~nm}$ emission is based on simple geometry, it is farther down the magnetic field line from the electron source region. The model profiles as implemented do have one issue, as explicitly noted in section 3, they do not include suprathermal electron flux losses to ambient background electrons. We did find that if we left out suprathermal electron fluxes below $20 \mathrm{eV}, 777.4 \mathrm{~nm}$ emission would be from more deeply penetrating electrons and the profile would be below that for $557.7 \mathrm{~nm}$, as the observations show. Since the emission profiles for $10-20 \mathrm{eV}$ are essentially the same, it is really only the electrons below $10 \mathrm{eV}$ that lead to $557.7 \mathrm{~nm}$ being the lowest altitude for all emission profiles. At these low energies $(<10 \mathrm{eV})$ we still have a competition between losses to the ambient electron gas (omitted), and $\mathrm{O}\left({ }^{1} \mathrm{D}\right), \mathrm{O}\left({ }^{1} \mathrm{~S}\right)$ and vibrational excitation of $\mathrm{N}_{2}$ all included. Qualitatively we know energy loss of suprathermal electron energy to ambient electrons is preferentially below $10 \mathrm{eV}$, but what can we say quantitatively?

Abreu and Carlson (1977) have published the impact of photoelectron energy losses to ambient electrons, as experienced at Arecibo (the same energy range as here), and compared their observed loss to that calculated from theory (Schunk and Hays 1971). We reproduce their Figure 8 (Abreu and Carlson 1977), as our Figure 6 here. We see their agreement between observation and theory was remarkable. Most importantly for our work here, is the impact on $557.7 \mathrm{~nm}$ emission of adding that loss of suprathermal electron flux below $10 \mathrm{eV}$. The impact: reduce the $557.7 \mathrm{~nm}$ emission on the bottom side by a factor exceeding half an order of magnitude; reduce the calculated bottom-side $557.7 \mathrm{~nm}$ emission to less than that calculated for 777.4 ; and restore $777.4 \mathrm{~nm}$ emission to being at a lower altitude than $557.7 \mathrm{~nm}$. It leads to a much reduced role of secondary electrons for impact excitation of bottom-side $557.7 \mathrm{~nm}$ profile.

For analysis of optical data, if we want to look at individual emission lines we have lost little, they are each instructive in their own way. If we want to combine $557.7 \mathrm{~nm}$ emissions with higher energy threshold emissions to 
derive a suprathermal electron spectra, we now have to work harder than previously generally realized. Losses of secondary electron impact excitation of bottom side $557.7 \mathrm{~nm}$ emissions must be factored into analysis.

In summary, to recap:

First, we have observed HF electron impact excited optical emissions at Arecibo from $630.0 \mathrm{~nm}, 557.7 \mathrm{~nm}$, and $777.4 \mathrm{~nm}$ wavelengths. In and of itself, the 777.4 emission is unequivocal proof that at Arecibo we had HF accelerated electron fluxes accelerated to energies $\sim 11 \mathrm{eV}$. Within the context of their persistence and the persistence of plasma line enhancements observed previously (Carlson et al. 1982), plus the experimental and theoretical evidence for spectral flatness across 10-20 eV (Carlson et al. 1982; Gurevich et al. 2000), we conclude that observation of $777.4 \mathrm{~nm}$ and $844.6 \mathrm{~nm}$ (Hysell et al. 2014) emissions $(10.74,10.99 \mathrm{eV})$ are a good surrogate for presence of HF electron fluxes in the ionizing range (13-19 eV).

Second, in this sense alone, observations of each of many individual wavelengths have been and continue to be of value to help guide theory of plasma physics and potential applications as e.g. production of artificial ionospheres.

Third, regarding spectra, to construct or guide theory to improved prediction of HF accelerated energy spectra based on energy integrals constrained by optical observations, further effort offers good value (e.g. Gurevich 2007 and many references therein). Gustavsson et al. (2005) used optical emissions to set parameters in a physics based model, but then returned in Gustavsson and Eliasson (2008) to notably improve realism of the findings by adding altitude dependencies of fluxes. Hysell et al (2014) introduced and applied a method to estimate the suprathermal electron population versus altitude and energy, during an F region HF ionospheric modification experiment, on the basis of observed emissions and an inversion method based on a variation of the classic Backus and Gilbert (1970) approach, including utilization of Green's functions to reduce the dimensionality of the problem. The nonparametric method was in contrast to the Gustavsson and Eliasson (2008) approach using airglow emissions to set the parameters of a physics-based electron acceleration model. They do a thorough listing of the competing crosssections, including $\mathrm{N}_{2}$ vibrational excitation essential to the composite electron impact cross-sections in the $1.5-5 \mathrm{eV}$ range (Itikawa 2006). The Hysell et al. (2014) work was motivated by not overly constricting derived spectra to input assumptions about a spectral shape from a theory still in development. Sergienko et al. (2012) have explored improvement in electron transport with a Monte Carlo method. Hysell et al. (2012) has likewise explored applying 
spectroscopy to estimate electron energy spectra and Eliasson et al. (2012) have done numerical modeling of artificial ionization layers at HAARP. Gurevich et al. (2004) have started from a theoretical derivation of HF accelerated electron fluxes noting optical emissions to which they should give rise. Work remains active to close this loop.

This work was similarly motivated by seeking understanding of limits and opportunities for more fruitful analysis of past and future data, and more robust was for future data collection, with the specific goals of combining multiple wavelength optical observations for improved realism of constraints on derived electron energy spectra.

Issues with $630.0 \mathrm{~nm}$ are well discussed in the literature (Rees and Roble 1975; Kalogerakis et al. 2009). In assessing the importance of $\mathrm{N}_{2}$ particularly of electron impact excitation of vibrational states (Itikawa 2006), it is important to keep track of the altitude dependence of number density of atomic oxygen vs molecular nitrogen, the ratio of which increase approximately an order of magnitude when going down in altitude from $\sim 300$ to $\sim 150 \mathrm{~km}$.

Here we document the importance of suprathermal electron loss to the ambient electron density in the F region. For our representative observations introduced here, we find that inclusion or omission of this loss term in the calculation makes the difference between the $557.7 \mathrm{~nm}$ emission being above or below the $777.4 \mathrm{~nm}$ emission peak. To make a more useful statement applicable at a general level, we highlight experiment and theory in agreement that an electron content of $\sim 2 \times 10^{13} \mathrm{~cm}^{-2}$ degrades the component of a flux of suprathermal electrons of energy $<\sim 10 \mathrm{eV}$, by about half an order of magnitude. This pertains to high nighttime electron densities, which are particularly attractive for HF heating experiments looking to work at higher HF frequencies for maximum HF power on target and electron acceleration.

For experimental work, it is thus important to measure/estimate electron density profiles if one wishes to use $630.0 \mathrm{and} /$ or $557.7 \mathrm{~nm}$ data in conjunction with higher energy threshold emissions (e.g. 777.4, 844.6, 427.8) to construct suprathermal electron energy spectra.

Specific techniques for design of experiments, data collection, and data reduction are highlighted here, both for general collection and including specific focus on Arecibo, where resumption of heating experiments is imminent, making these specific findings timely.

It is worth noting that from calculations tracking the cascade of energy from above $40 \mathrm{eV}$, it appears that the number of $\mathrm{eV}$ per ion pair produced is closer to $25 \mathrm{eV}$ per ion pair in the $\mathrm{F}$ region than the conventionally quoted nominal rule of thumb of $35 \mathrm{eV} /$ ionization pair (Rees and Roble 1986), which is more associated with E region 
aurora. This distinction may be of interest more generally for work on planetary atmospheres. In that community, Simon et al. (2011) treat this question in detail for five planetary atmospheres, and Fox et al. (2008) have delved deeper into tracing energy flow and deposition for other atmospheres.

We should point out that here in closing, that by design, the altitude of the source electrons in these calculations was defined as being held fixed, so the program was not intended to track a downward motion of an artificial ionization layer were such motion to occur as at HAARP (Pedersen et al. 2009, 2010). A program to track downward descent of any artificial ionization layer formed, would require full transport tracking of ambient background electrons through the thermosphere, in contrast to just the supra-thermal component discussed here.

\section{Conclusions}

The observations we present here were at several wavelengths to see how comparison of observation at different energy thresholds could illuminate knowledge of the spectra. This much is not new, the field has even moved on to as many as five wavelengths (e.g. Hysell 2014) to pursue such goals. What is new from our analysis here, including comparison of observations with aeronomical model runs (Carlson et al. 1982), sheds new light on past and future data collection goals, and analysis techniques.

The three most important conclusions we draw based on this work are:

1. We have shown that inclusion of suprathermal electron energy loss for energies below $10 \mathrm{eV}$, is an important consideration to include when the goal of the research is to combine observation of $557.7 \mathrm{~nm}$ emissions with higher energy threshold emissions (e.g. 777.4, 844.6, 427.8, etc.) in order to estimate or experimentally constrain/guide future theory and modeling of HF accelerated suprathermal electron fluxes. This particularly relates to issues with HF production of artificial ionospheres.

2. For past and future data: The altitude differences in observed electron impact excited optical emissions can make a valuable observational contribution to and constraint on understanding the essential transport part of the overall interpretation. However to realize this potential, account needs to be taken of losses of the component of electron flux below $10 \mathrm{eV}$, where electron densities/content can be a reasonable fraction of daytime values.

3. For future observations: (a) The geometry of the Arecibo magnetic field makes direct observations overhead the HF heater valuable to trace from the acceleration source altitude to the different center-of-gravity stopping 
altitudes for different optical wavelengths emissions. This observational differentiation is of value to interpretation. It significantly mitigates the observational loss when side-looking optics is absent, and compliments the added value when present and (b) one should make coincident measurements of the altitude profile of electron density.

Acknowledgements We wish to acknowledge essential support from the Air Force Office of Scientific Research (AFOSR), under Grant FA9550-11-1-0236. Appreciation is extended to the Arecibo Observatory staff for its always helpful support to visitors and to its research mission. This data was collected on a field trip with a dear friend and colleague, now sorely missed by many, Ed Weber. Peter Ning provided invaluable support to early optical data processing. George Mantas briefly interrupted a pleasant retirement to resuscitate software for a next generation at USU.

\section{References:}

V.J. Abreu, H.C. Carlson, J. Geophys. Res., 82, 1017-1023 (1977)

G. Backus, F. Gilbert, Philos. Trans. R. Soc. London, Ser. A., 266, 123-192 (1970)

P.A. Bernhardt, C.A. Tepley, L.M. Duncan, J. Geophys. Res., 94, 9071-9092 (1989)

N.F. Blagoveshchenskaya, H.C. Carlson, V.A. Kornienko, T.D. Borisova, M.T. Rietveld, T.K. Yeoman, A. Brekke, Ann. Geophys., 27, 131-145 (2009)

H.C. Carlson, Artificial ionosphere-creation using high power HF transmitters, AFGL1987/ILIR7L, AFGL(PL/CAG), Hanscom AFB, MA, 01731 (1987)

H.C. Carlson, Adv. Space Res., 13, 1015-1024 (1993)

H.C. Carlson, Proceedings HG3 Ionospheric modification by high power radio waves: coupling of plasma processes, URSI General Assembly, Lille France (1996)

H.C. Carlson, V.B. Wickwar, G.P. Manthas, J. Atmos. Terr. Phys., 44, 1089-1100 (1982)

H.C. Carlson, W.E. Gordon, R.L. Showen, J. Geophys. Res., 77, 1242-1250 (1972)

F.T. Djuth, T.R. Pedersen, E.A. Gerken, P.A. Bernhardt, C.A. Selcher, W.A. Bristow, J.H. Kosch, Phys. Rev. Lett., 94, $125001(2005)$

B. Eliasson, X. Shao, G.M. Milikh, E.V. Mishin, K.D. Papadopoulos, J. Geophys. Res., 117, A10321, (2012)

J.A. Fejer, Geophys Res. Lett., 4(7), 289-290 (1977)

J.A. Fejer, Rev. Geophys.1, 7, 135-153 (1979)

P.A. Fialer, Radio Sci., 9 (1974)

J.L. Fox, M.I. Galand, R.E. Johnson, Space Sci. Rev., 136; 3-62 (2008)

Y. Gong, Z. Qihou, Z. Shaodong, N. Aponte, M. Sulzer, S. Gonzalez, J. Geophys. Res., 117, A08331 (2012)

W.E. Gordon, R.L. Showen, H.C. Carlson, J. Geophys. Res., 76, 7808-7813 (1971)

A.V. Gurevich, Usp. Fizicheskikh Nauk., 177(11), 1145-1177 (2007)

A.V. Gurevich, Y.A. Dimant, G.M. Milikh, V.V. Vaskov, J. Atmos. Terr. Phys., 47, 1057-1070 (1985)

A.V. Gurevich, H.C. Carlson, G.M. Milikh, K.P. Zybin, F.T. Djuth, K. Groves, Geophy. Res. Lett., 27, 2462-2464 (2000)

A.V. Gurevich, H.C. Carlson, K.P. Zybin, Phys. Lett. A., (2001)

A.V. Gurevich, K.P. Zybin, H.C. Carlson, T.Pedersen, Phys. Lett. A, 305, 264-274 (2002)

A.V. Gurevich, H.C. Carlson, Y.V. Medvedev, K.P. Zybin, Plasma Phys. Rep., 30(12), 995-1005 (2004)

A.V. Gurevich, K.P. Zybin, H.C. Carlson, Radiophys. Quantum Electron. (Engl. Transl.), 48, 9 (2005)

B. Gustavsson, et al., Ann. Geophys., 23, 1747-1754 (2005)

B. Gustavsson, B. Eliasson, J. Geophys. Res., 113, A08319 (2008)

J.C. Haslett, L.R. Megill, Radio Sci., 9, 1005-1019 (1974)

D.L. Hysell, R.H. Varney, M.N. Vlasov, E.Nossa, B. Watkins, T. Pedersen J.D. Huba, J. Geophys. Res., 117, 
A02317 (2012)

D.L. Hysell, R.J. Miceli, E.A. Kendall, N.M. Schlatter, R.H. Varney, B.J. Watkins, T.R. Pedersen, P.A. Bernhardt, J.D. Huba, J. Geophys. Res. Space Phys., 119, 2038-2045 (2014)

Y. Itikawa, J. Phys. Chem. Ref. Data, 35, No 1 (2006)

K.S. Kalogerakis, T.G. Slanger, E.A. Kendall, T.R. Pedersen, M.J. Kosch, B. Gustavsson, M.T. Rietveld, Ann. Geophys., 27, 2183-2189 (2009)

M.J. Kosch, M.T. Rietveld, T. Hagfors, T.B. Leyser, Geophys. Res. Lett., 27, 2817-2820 (2000)

G.P. Mantas, H.C. Carlson, C. LaHoz, J. Geophys. Res., 86, 561-574 (1981)

C.K. Mutiso, J.M. Hughes, G.G. Sivjee, T.Pedersen, B. Gustavsson, M.J. Kosch, Geophys. Res. Lett., 35, L14103 (2008)

T.R. Pedersen, H.C. Carlson, Radio Sci., 36, 1013-1026 (2001)

T.R. Pedersen, B. Gustavsson, E. Mishin, E. MacKenzie, H.C. Carlson, M. Starks, T. Mills, Geophys. Res. Lett., 36, L18107 (2009)

T. Pedersen, B. Gustavsson, E. Mishin, E. Kendall, T. Mills, H.C. Carlson, A.L. Snyder, Creation of artificial ionospheric layers using high-power HF waves, Geophys. Res. Lett., 37, L02106 (2010)

F.W. Perkins, C. Oberman, E.J. Valeo, J. Geophys. Res., 79, 1478-1496 (1974)

M.H. Rees, R.G. Roble, Rev. Geophys., 13, 201-242 (1975)

M.H. Rees, R.G. Roble, Can. J. Phys., 64, 1608 (1986)

G. Rose, B. Grandal, E. Neske, W. Ott, K. Spenner, J. Holtet, K. Mâseide, J. Trøim, J. Geophys. Res., 90, 2851$2860(1985)$

R.W. Schunk, P.B. Hays, Planet. Space Sci., 19, 113 (1971)

T. Sergienko, B. Gustavsson, U. Bringstrom, K. Axelsson, Geophys., 30, 885-895 (2012)

W. Simon, C., G. Gronoff, J. Lilensten, H. Ménager, M. Barthélemy, Ann. Geophys., 29, 187-195 (2011)

D.P. Sipler, M.A. Biondi, J. Geophys. Res., 77, 6202-6212 (1972)

D.J. Strickland, J.R. Jasperse, J.A. Whalen, J. Geophys. Res., 88, 8051 (1983)

W.F. Utlaut, R. Cohen, Science, 174, 245-254 (1971)

J. Weinstock, Radio Sci., 9, 1085-1087 (1974) 


\section{Figure Captions}

Fig. 1 Original data Arecibo April 20, 1988, for $630.0 \mathrm{~nm}$ emission and surrounding background, in single frame image, with no data processing beyond increasing degrees of smoothing of adjacent pixels, to reduce noise fluctuations in image. Clear contours stand out well against background with no further subtraction

Fig. 2a Arecibo observations April 20, 1988, $15 \mathrm{~s}$ integrations, in projection of HF electron excited optical emission contours (derived as in Figure 1) for $630.0 \mathrm{~nm}$ (in red), $557.7 \mathrm{~nm}$ (in green) and $777.4 \mathrm{~nm}$ (in violet), horizontally aligned in the magnetic meridian plane, then moved vertically along the magnetic field line over Arecibo Observatory. The vertical scale is about half a neutral oxygen scale height (tens of $\mathrm{km}$ )

Fig. 2b HAARP observations (Pedersen et al, 2010) of $557.7 \mathrm{~nm}$ images for 210 s combined by a tomographic algorithm, giving a cross-cut of the optical volume emission rate in the magnetic meridian plane. The HAARP magnetic field line and contours of nominal transmitter power in percent relative to peak power at $230 \mathrm{~km}$ altitude are superimposed on the tomographic map

Fig. 3 Relative fluxes of up-going and down-going suprathermal electrons at several altitudes in the observation region were derived from a uniform production rate from zero to $20 \mathrm{eV}$ electrons, all originating at $275 \mathrm{~km}$. This figure shows their decomposition by energy of the up-going and down-going suprathermal electron fluxes at 256 and $266 \mathrm{~km}$. The calculations partition electrons into $2 \mathrm{eV}$ segments of the spectrum at $275 \mathrm{~km}$, and were traced to other energy ranges at lower or higher altitudes. The right-hand figure shows the downward flux at the two altitudes. The left-hand figure shows the upward flux obtained from electrons backscattered at the indicated and lower altitudes (Carlson et al. 1982)

Fig. 4 Shows the results for a single thin altitude source slab of HF accelerated electrons at three altitudes, one at which the thermosphere is: very optically thin $(982 \mathrm{~km})$ far right hand side, intermediate $(505 \mathrm{~m})$ center, one at a depth at which the thermosphere is optically thick $(254 \mathrm{~km})$ left hand side of figure, and one intermediate $(505 \mathrm{~km})$ in the central column of the figure. The only difference between the top and bottom row is the flux scale, to visualize a dynamic range of a factor of 50 in suprathermal flux. This shows penetration depth of composite electron flux including all primary and secondary electrons

Fig. 5a Shows results from calculations with the same model as for Figure 4 for electron impact excitation and ionization, but in the form of altitude profiles of Volume Emission Rate (photons $\mathrm{m}^{-3} \mathrm{~s}^{-1}$ ) for $557.7 \mathrm{~nm}$ and 777.4 $\mathrm{nm}$, and $\mathrm{O}^{+}$ionizations $\mathrm{m}^{-3} \mathrm{~s}^{-1}$, all for atomic oxygen (thresholds respectively of $\sim 4.2,10.7,13.6 \mathrm{eV}$ ). While Figure 4 was for the sum of all primary and secondary electrons, Figure 5 shows plots for the primaries only in $10 \mathrm{eV}$ wide bins $(\mathrm{X}$ to $\mathrm{X}+10 \mathrm{eV}$ ), one at a time (still with flux flat between 1-100 eV), and in addition also plots the same scale the sum of these primary electrons plus all secondary electrons $(1$ to $\mathrm{X} \mathrm{eV})$ which that $10 \mathrm{eV}$ wide bin produced at and below its energy interval. Figure 5a is for the optically thin case source electrons at $982 \mathrm{~km}$.

Fig. 5b Same as Figure 5a but for the optically thick case, source electrons at $254 \mathrm{~km}$

Fig. 6 (a) Differential particle fluxes obtained by degrading the experimental spectrum shown in and Abreu and Carlson (1977) Figures 5a and $8 \mathrm{~b}$ at $1.0 \times 10^{13} \mathrm{~cm}^{-2}$ using the Schunk and Hays (1971) energy loss expression; (b) The observed differential particle fluxes for matched conditions 

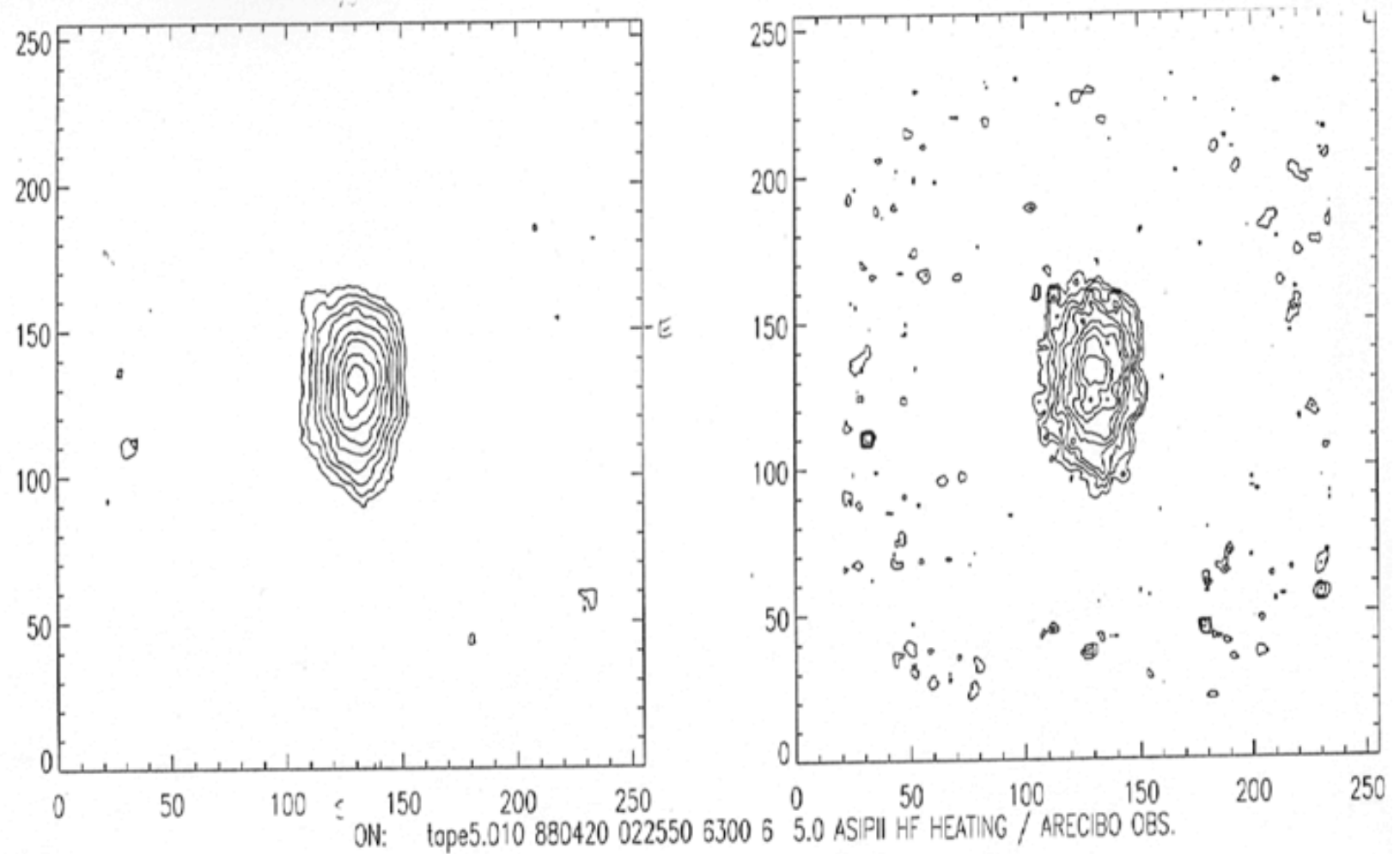

OFF: tope5.016 880420 022750630065.0 ASIP|| HF HEATING / ARECIBO OBS.
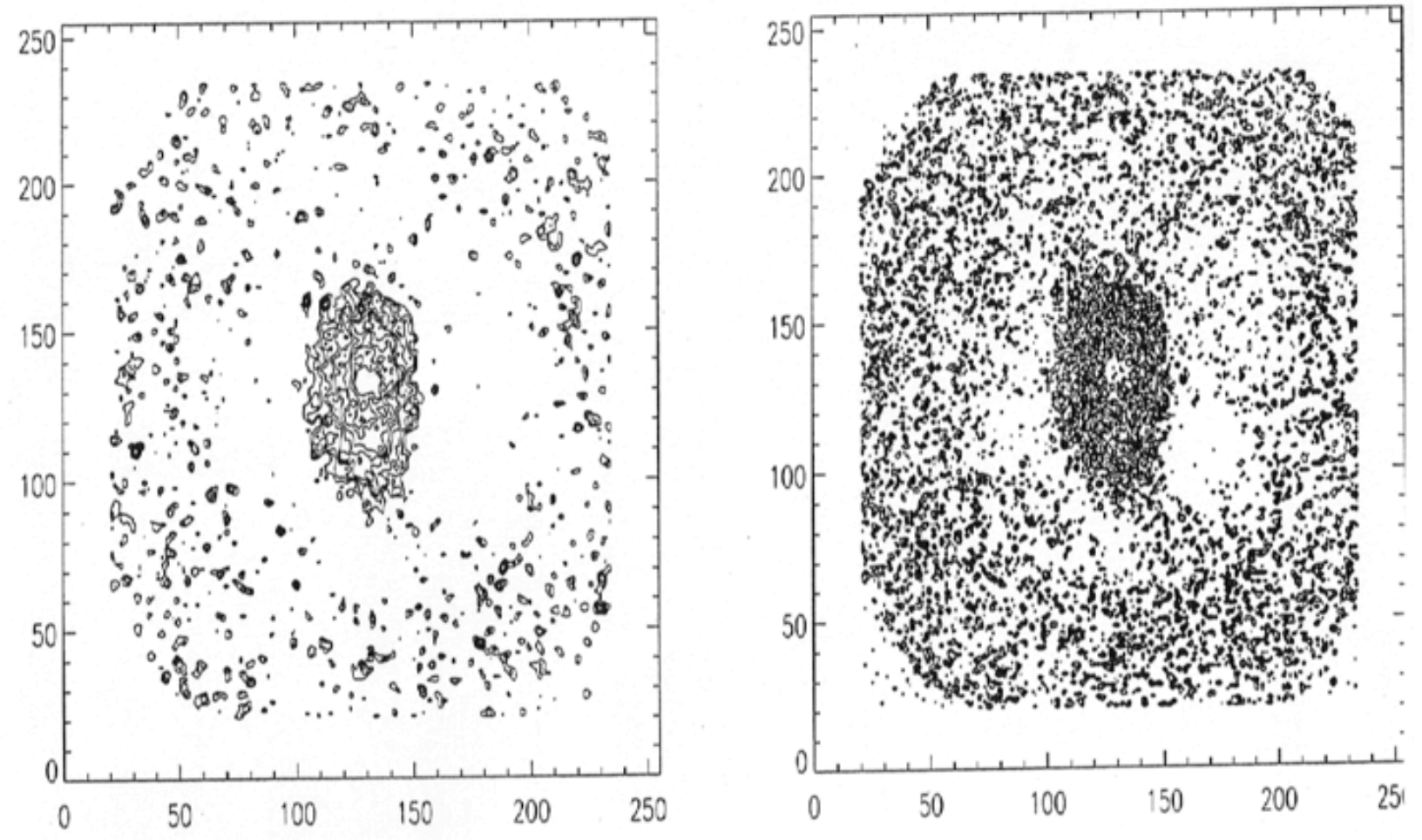

Figure 1 


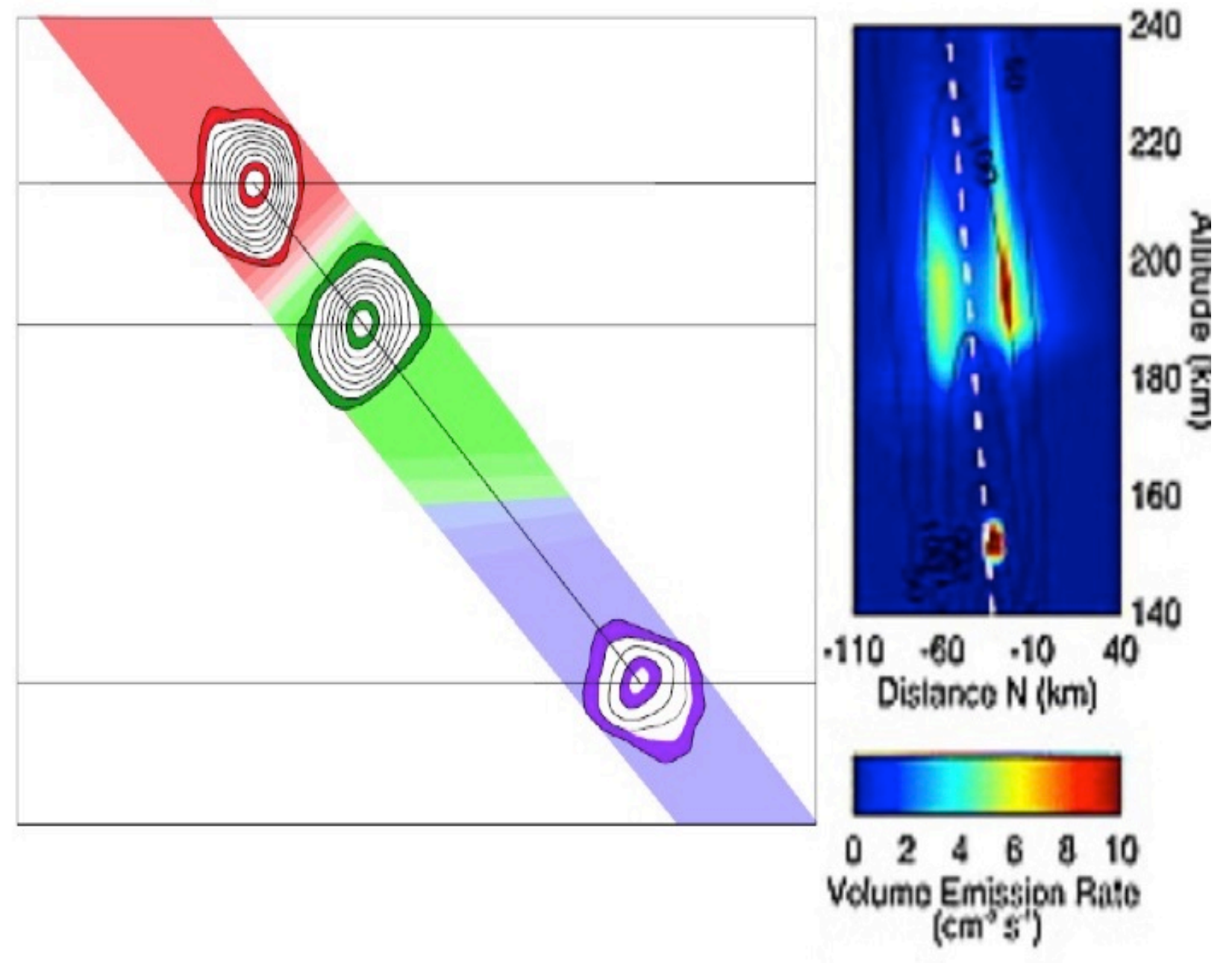

figure 2 


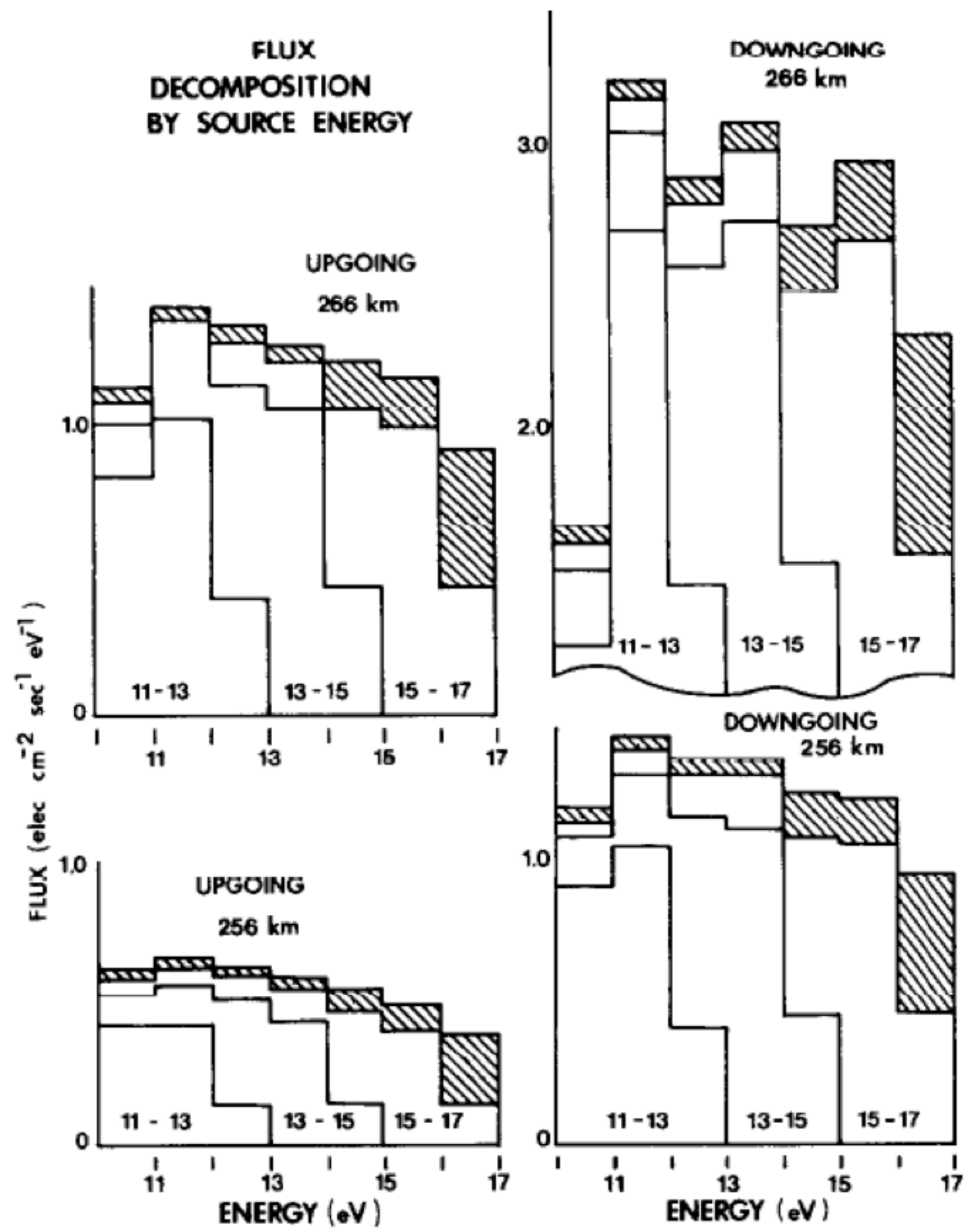

figure 3 
Steady State Flux (input of full primaries 1-100ev)
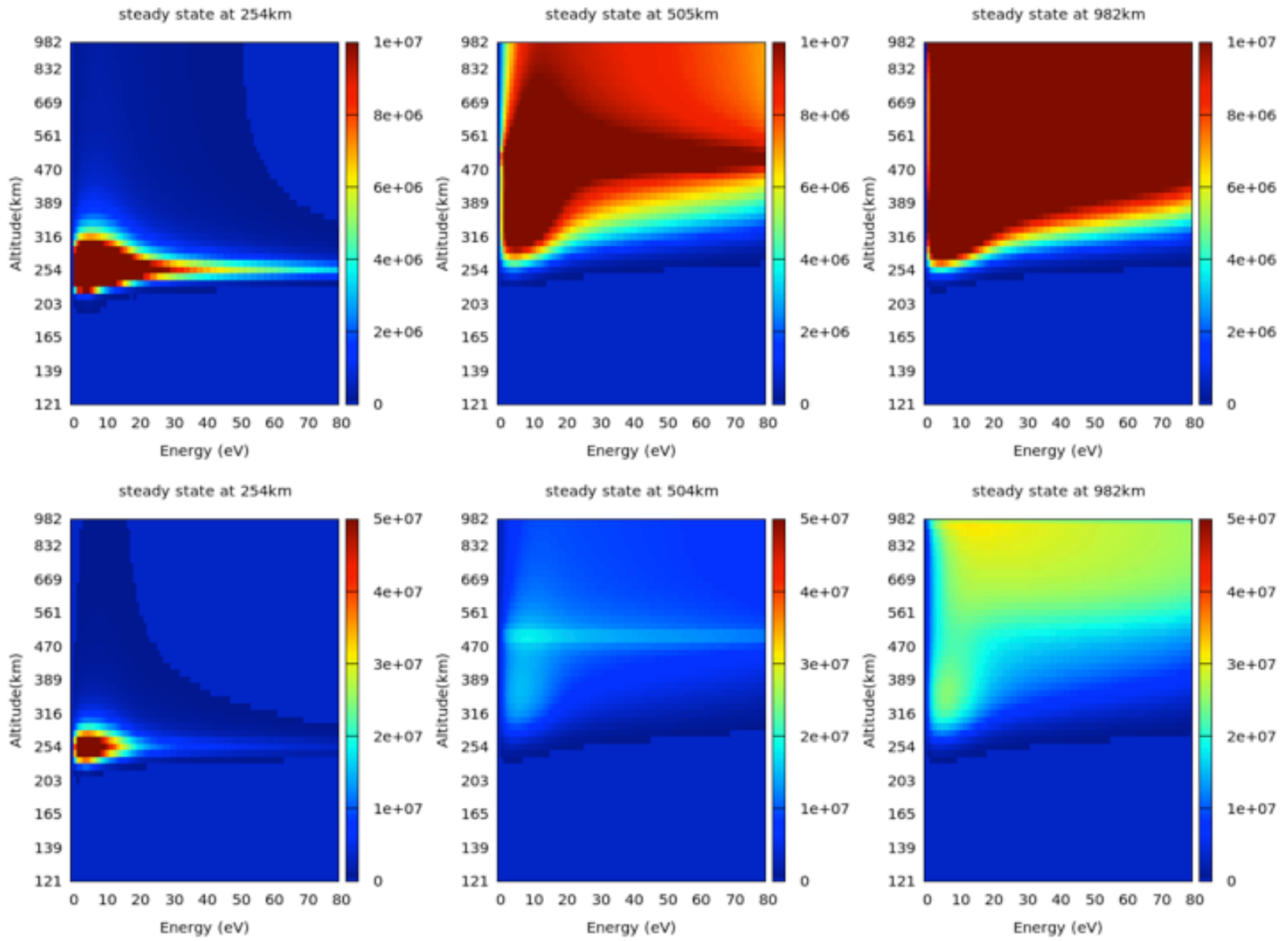

figure 4 
$982 \mathrm{~km}$ (full primary inputs, steady state flux binned for optical emission calculation)

$41-50 \mathrm{eV}$ at $982 \mathrm{~km}$

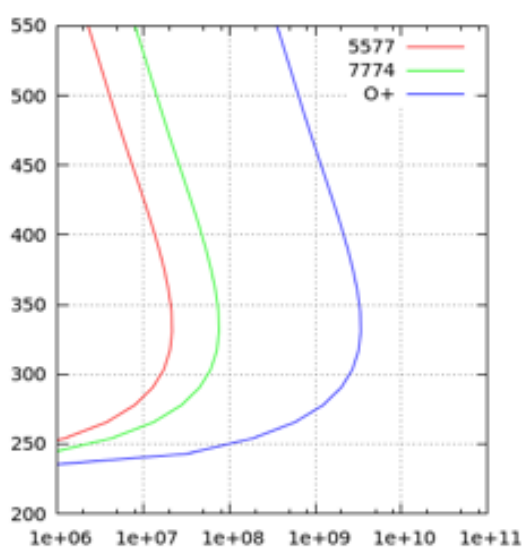

$61-70 \mathrm{eV}$ at $982 \mathrm{~km}$

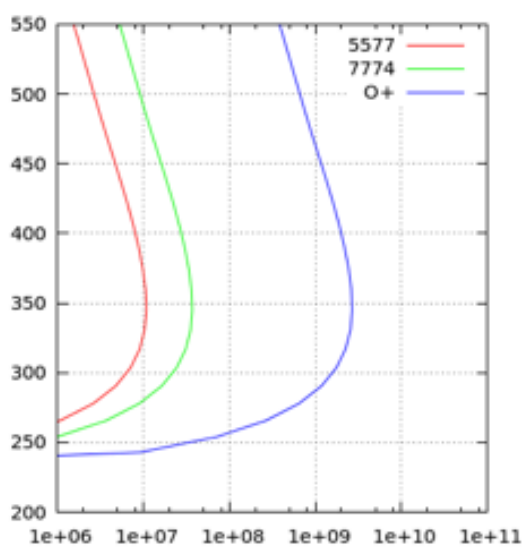

$51-60 \mathrm{eV}$ at $982 \mathrm{~km}$

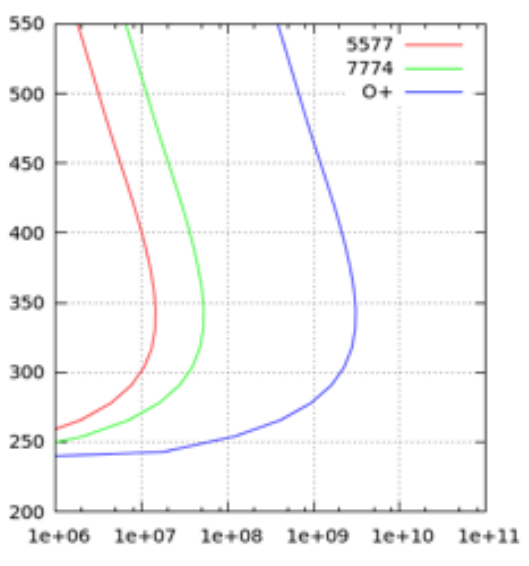

$71-80 \mathrm{eV}$ at $982 \mathrm{~km}$

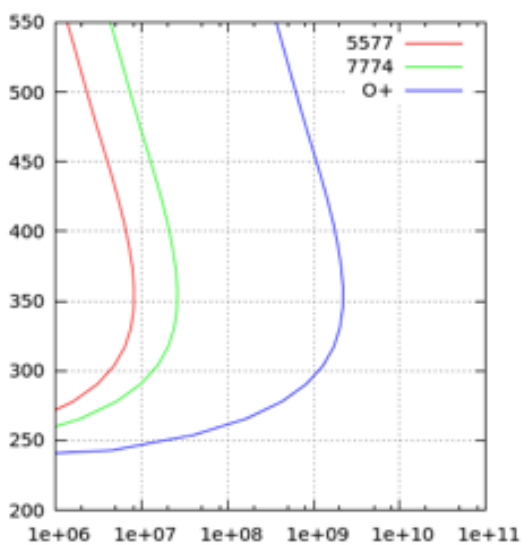

$1-60 \mathrm{eV}$ at $982 \mathrm{~km}$

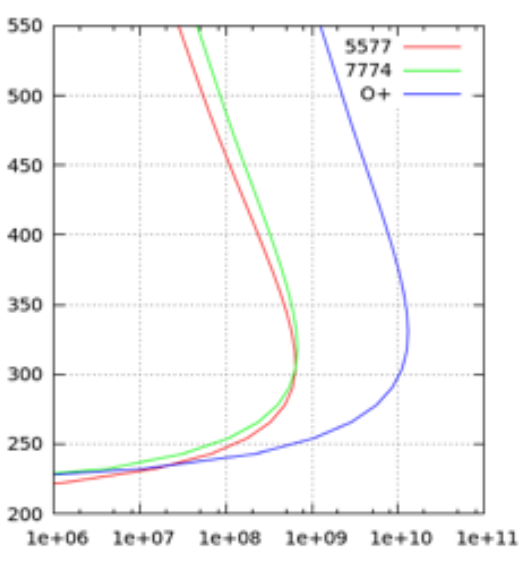

$1-80 \mathrm{eV}$ at $982 \mathrm{~km}$

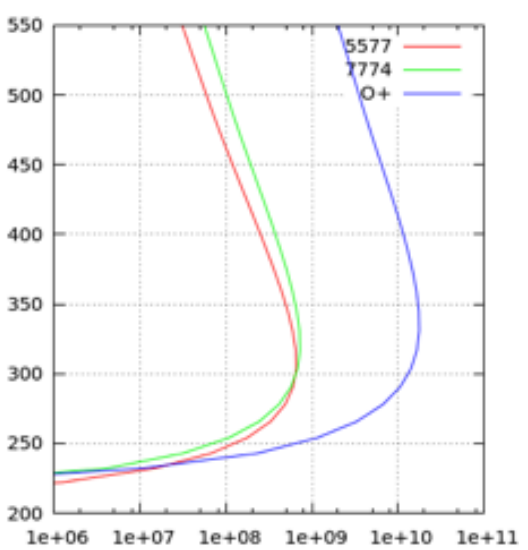

Figure 5a1 
$982 \mathrm{~km}$ (full primary inputs, steady state flux binned for optical emission calculation)

$1-10 \mathrm{eV}$ at $982 \mathrm{~km}$

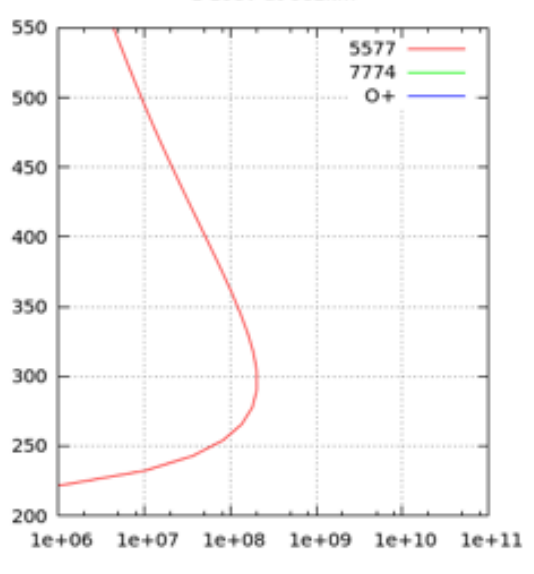

$21-30 \mathrm{eV}$ at $982 \mathrm{~km}$

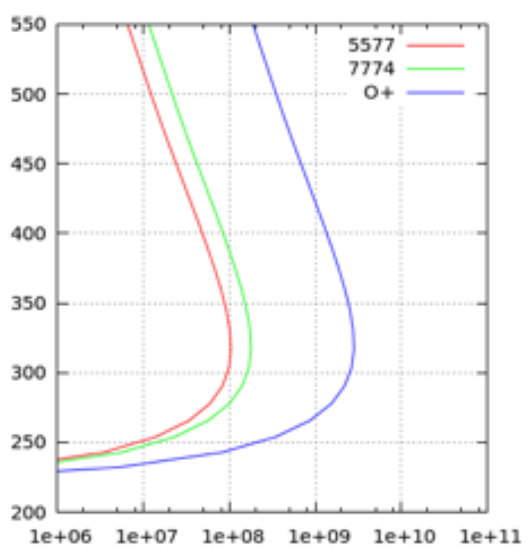

$11-20 \mathrm{eV}$ at $982 \mathrm{~km}$

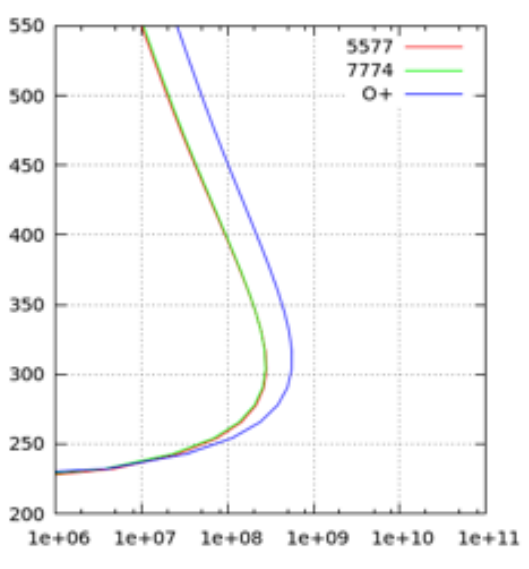

$31-40 \mathrm{eV}$ at $982 \mathrm{~km}$

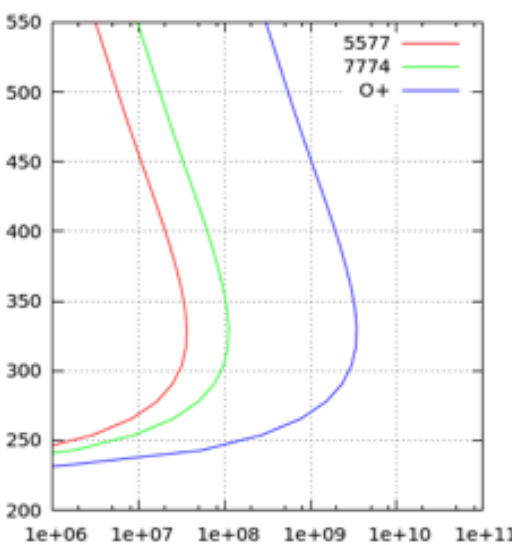

$1-20 \mathrm{eV}$ at $982 \mathrm{~km}$

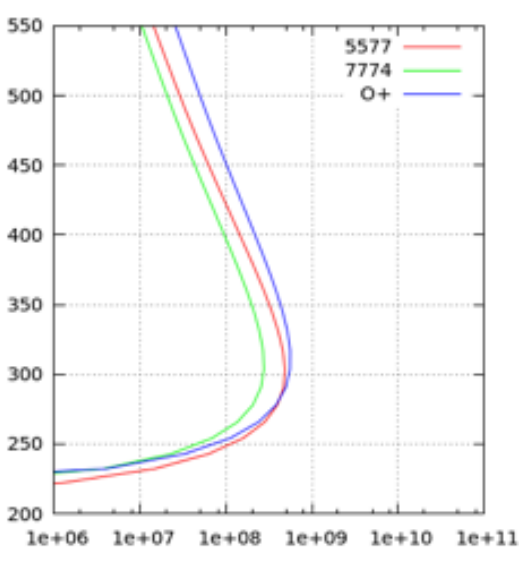

$1-40 \mathrm{eV}$ at $982 \mathrm{~km}$

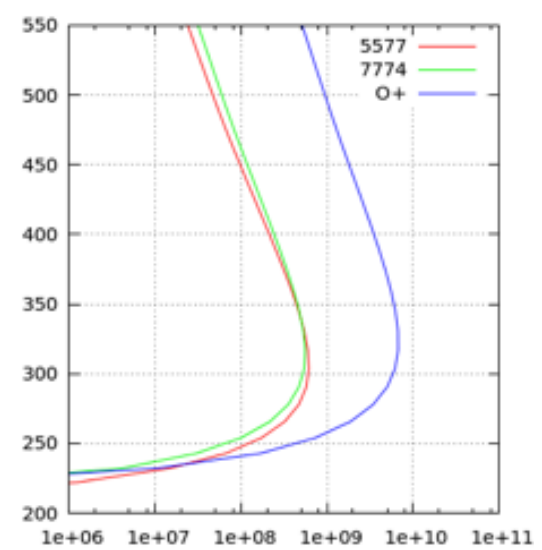

Figure $5 \mathrm{a} 2$ 
$254 \mathrm{~km}$ (full primary inputs, steady state flux binned for optical emission calculation)

$41-50 \mathrm{eV}$ at $254 \mathrm{~km}$

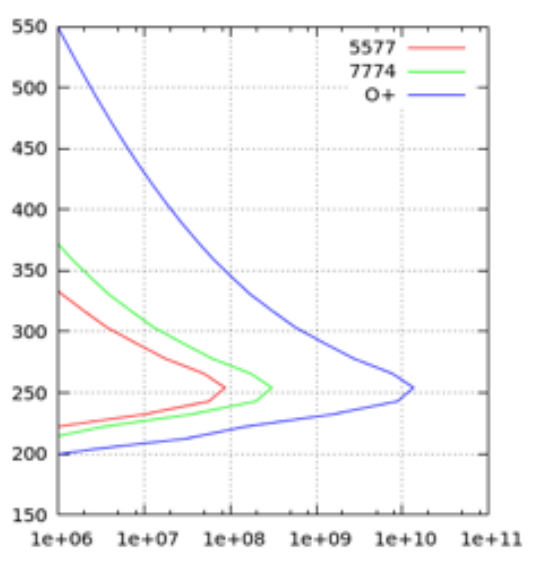

$61-70 \mathrm{eV}$ at $254 \mathrm{~km}$

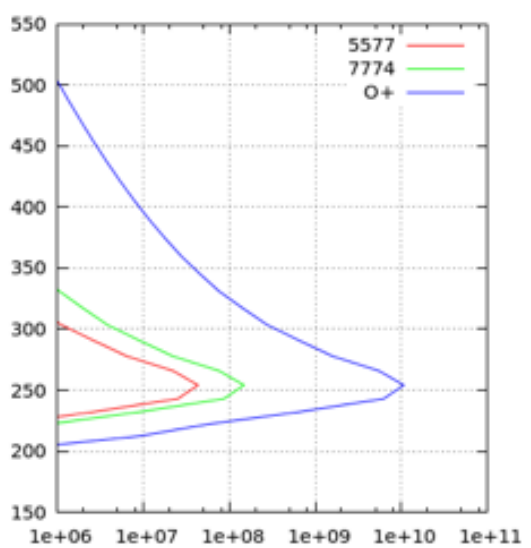

$51-60 \mathrm{eV}$ at $254 \mathrm{~km}$

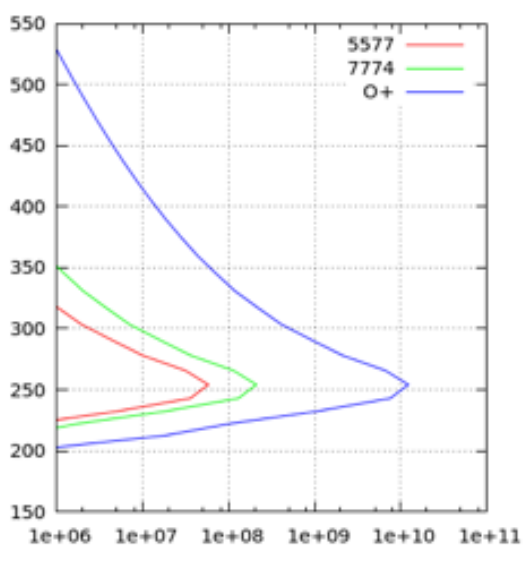

$71-80 \mathrm{eV}$ at $254 \mathrm{~km}$

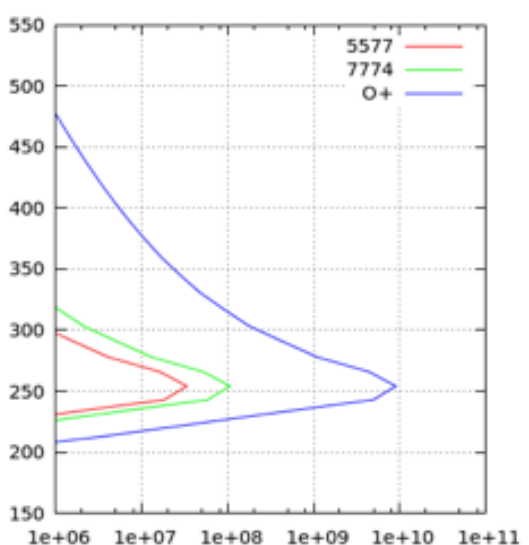

$1-60 \mathrm{eV}$ at $254 \mathrm{~km}$

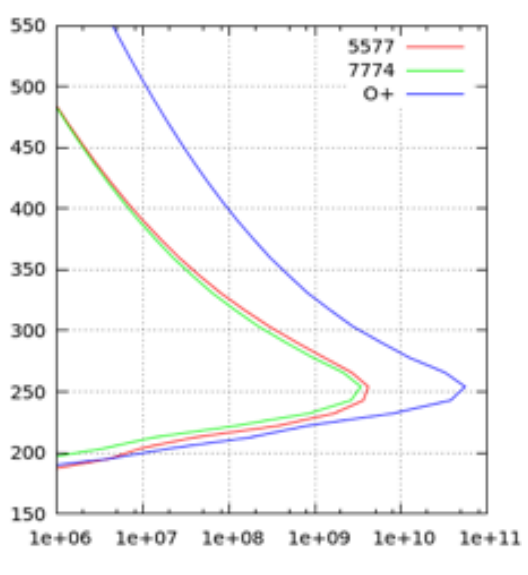

$1-80 \mathrm{eV}$ at $254 \mathrm{~km}$

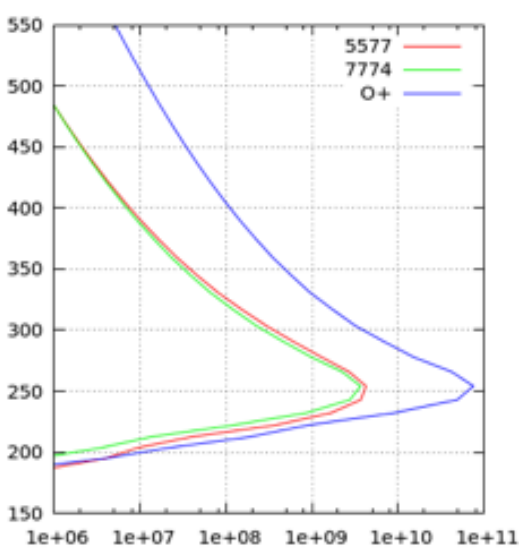

Figure 5b1 
$254 \mathrm{~km}$ (full primary inputs, steady state flux binned for optical emission calculation)

$1-10 \mathrm{eV}$ at $254 \mathrm{~km}$

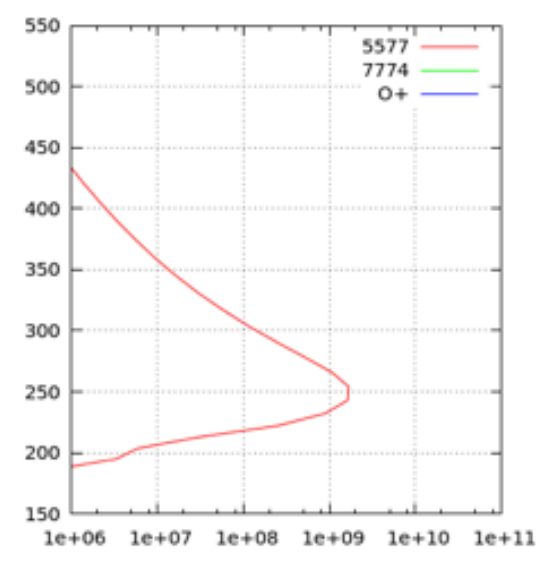

$21-30 \mathrm{eV}$ at $254 \mathrm{~km}$

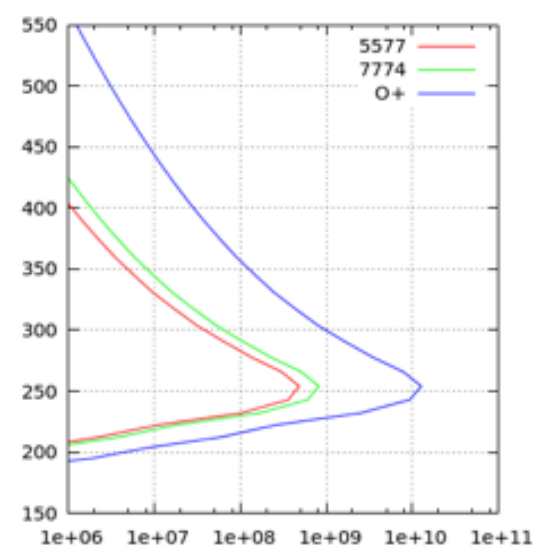

$11-20 \mathrm{eV}$ at $254 \mathrm{~km}$

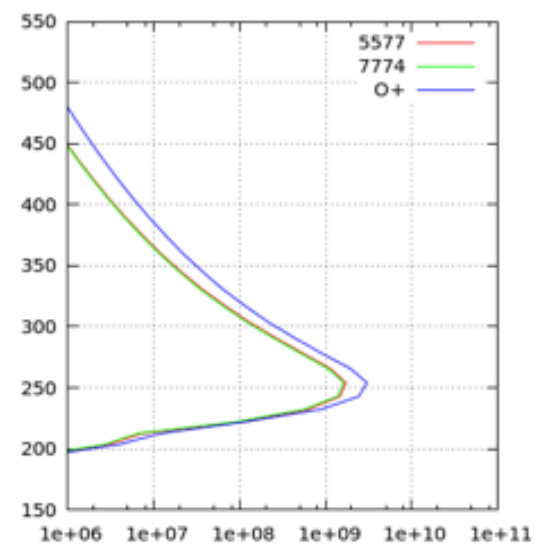

$31-40 \mathrm{eV}$ at $254 \mathrm{~km}$

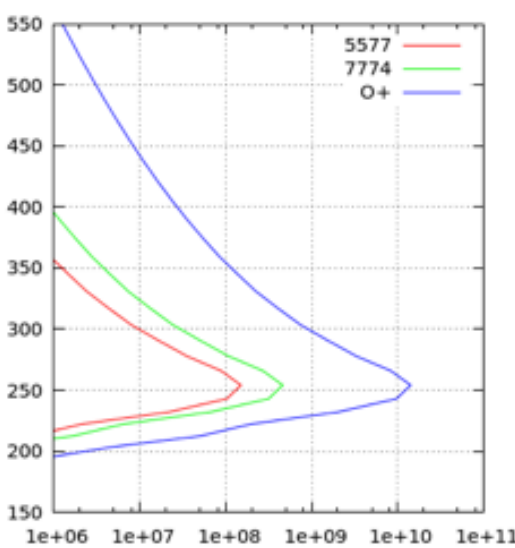

$1-20 \mathrm{eV}$ at $254 \mathrm{~km}$

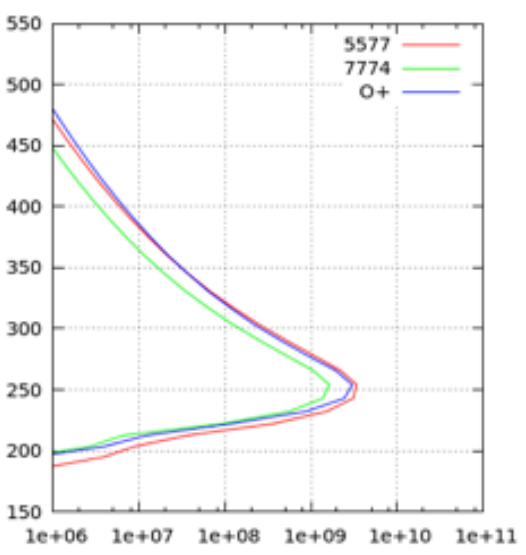

$1-40 \mathrm{eV}$ at $254 \mathrm{~km}$

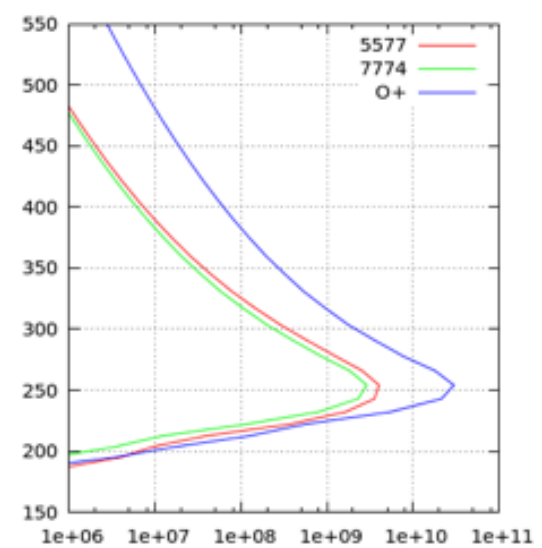

Figure $5 \mathrm{~b} 2$

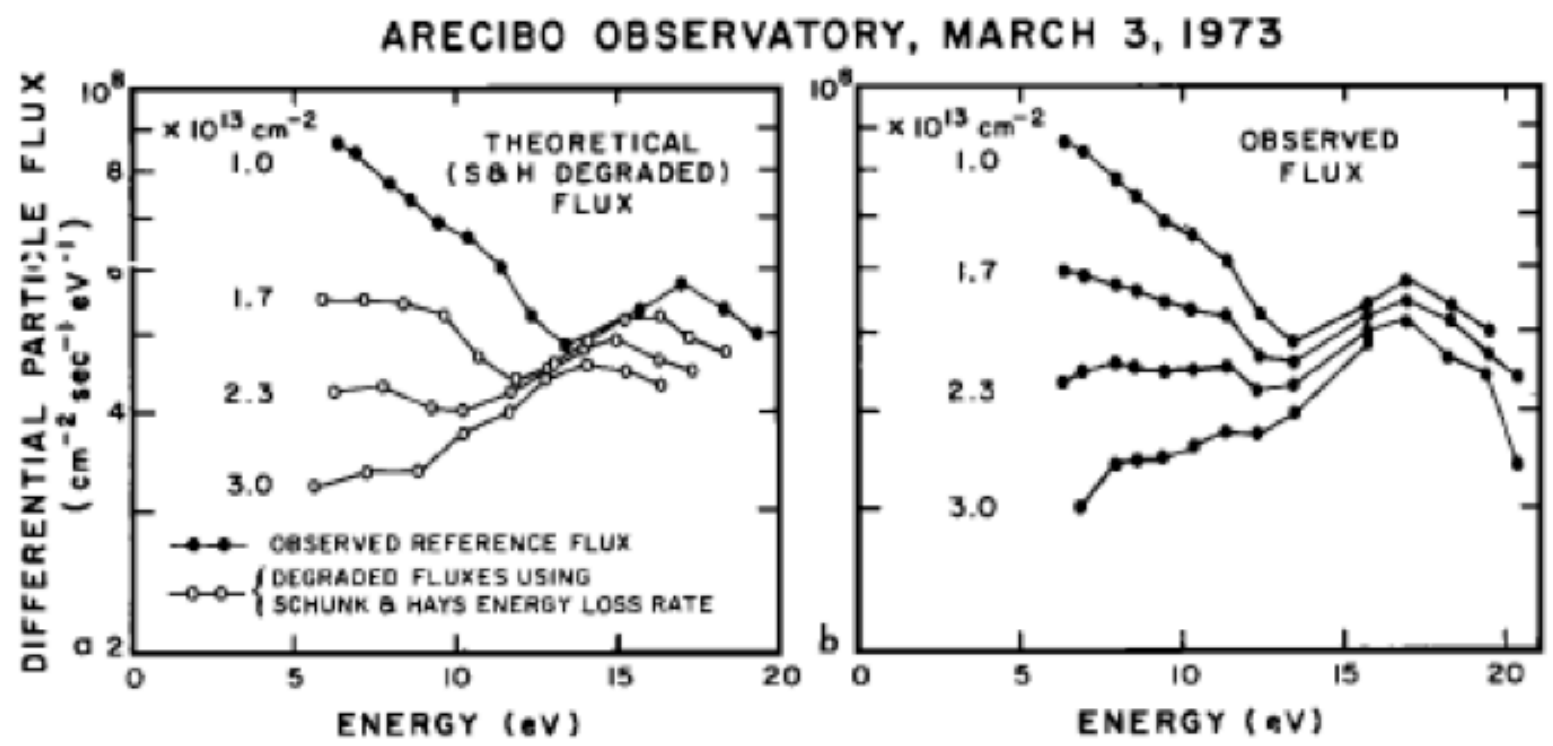

Figure 6 